\title{
Comportamiento logístico de las empresas de manufactura en Querétaro, México
}

\author{
Ovidio GonZÁLEZ-GÓMEZ* \\ Rossana NIETO-LÓPEZ**
}

\begin{abstract}
Resumen
Esta investigación examina la relación entre tipo de procesos productivos y prácticas logísticas en un área mexicana de reciente industrialización: el corredor industrial San Juan del Río en el estado de Querétaro. Se considera que el alcance de las relaciones comerciales define, junto con las formas de organización de la producción, el tipo de práctica logística asumida por las plantas industriales.
\end{abstract}

Palabras clave: Querétaro, logística, manufactura, procesos productivos, industrialización.

\begin{abstract}
This paper examines the relationship between production processes and logistic practices in an area of recent industrialisation in Mexico: the San Juan del Rí industrial corridor in Queretaro State. It is considered that the reach of commercial relationships together with the different forms of production organisation, define the kind of logistic practice adopted by different industrial plants.
\end{abstract}

Keywords: Queretaro, logistics, manufacture, production processes, industrialisation.

\footnotetext{
* Facultad de Ingeniería de la Universidad Autónoma de Querétaro, México. Correo-e: ovidio@uaq.mx.

** Profesora en El Colegio de Bachilleres del estado de Querétaro, México. Correo-e: ronielo@hotmail.com.
} 


\section{Introducción $^{1}$}

El proceso globalizador, como quiera que se le defina, ha cambiado gran parte de los comportamientos productivos y comerciales en la mayoría de las actividades económicas, y el transporte no es la excepción. Actividades manufactureras líderes, como las industrias automotriz y microelectrónica, muestran hoy en día redes casi mundiales que permiten la reconstitución de un sinnúmero de fragmentos productivos, muy vinculados funcionalmente, pero disgregados en el territorio mundializado. Esta tendencia difundida en el mundo ejerce presión sobre el traslado y distribución física de la mercancía.

Los cambios en el tipo de organización de la producción (del fordismo a la especialización flexible), la homogeneización de los patrones de consumo y la relocalización de los procesos productivos que ocurrieron a lo largo de las tres últimas décadas, llevaron al surgimiento de la logística, no sólo como un campo de estudio sino, sobre todo, como una práctica socialmente aceptada y como una actividad económica dentro de la organización de los procesos productivos cuya importancia en términos económicos es indiscutible, pues engloba la serie de actividades que reconstituyen el proceso productivo y que convierten de forma competitiva el producto en mercancía en el mercado mundial, y, además, en cuya ausencia no se cerraría el ciclo de rotación de capital a escala mundial.

La mayoría de los enfoques sobre el concepto de logística lo definen en relación con el último eslabón de la cadena entre producción y consumo; siguiendo la definición del Consejo de Administración Logística de Estados Unidos (CLM, por sus siglas en inglés): "Logística es el proceso de planeación, implementación y control eficiente y eficaz de flujos y almacenamiento de bienes, servicios e información relacionada, desde el punto de origen al punto de consumo" (Bowersox y Closs, 1996).

En la práctica, sin embargo, a lo largo de las últimas tres décadas los comercios intrafirma e intraindustria han crecido de forma sostenida como resultado de la fragmentación de los procesos productivos y de la relocalización de partes de la cadena productiva (Fröbel et al., 1977; Piore y Sabel, 1984), ya sea hacia otros países centrales industrializados o hacia países periféricos

\footnotetext{
${ }^{1}$ Este trabajo es un resultado parcial de la investigación: Industrialización y mercados segmentados de transporte en el estado de Querétaro, financiada por el Sistema de Investigación Miguel Hidalgo (SIHGO) y el Conacyt.
} 
(Wallerstein, 1979). Así, la mayor parte de la mercancía en tránsito pertenece a los eslabones productivos intermedios y, por lo tanto, la logística se encuentra más en el ámbito de la administración del proceso productivo, en términos estrictos (Tixier et al., 1988). Si bien los costos del traslado de la mercancía se han incrementado en términos absolutos, su participación relativa en los precios del producto final ha decrecido notablemente, $y$, sobre todo, la posibilidad de utilizar mano de obra barata en prácticamente cualquier parte del mundo, modifica las estrategias productivas hacia un análisis de la estructura de costos del proceso productivo en su totalidad y no atendiendo a, por ejemplo, los diferenciales en los fletes exclusivamente.

El análisis sobre el concepto de logística, o incluso la conveniencia del concepto centrado en la demanda final (el consumo), o en la organización industrial relacionada con la esfera de la producción, quedan fuera del alcance de este artículo. Pero se considera importante aquí explorar algunos de los comportamientos reales relacionados con las plantas manufactureras en lo que corresponde a la manera en que estos actores económicos resuelven el posicionamiento espacio-temporal de la mercancía, bien sea de consumo final o productivo.

Como resultado del surgimiento de la logística, han aparecido nuevos actores que resuelven las necesidades de esas redes productivas y comerciales de escala casi mundial. Agentes de carga nacionales internacionales y multimodales, expertos logísticos y otros más han ganado lugar en el movimiento de la mercancía en tiempo y espacio.

Mientras que la estructura industrial en México ha sido estudiada en forma concienzuda, existen sólo algunos estudios empíricos relacionados con las prácticas de transportación y logísticas de las firmas manufactureras (Antún, 1995). La decisión de las firmas manufactureras sobre la forma de transportación es parte de las prácticas logísticas que, a su vez, se consideran en esta investigación como parte de la estrategia logística de la empresa.

El enfoque aquí seguido se basa en la premisa de que la estrategia logística se expresa claramente en la contratación de las empresas de transporte de acuerdo con un conjunto de atributos considerados decisivos, y esos requerimientos se reflejan en la forma en que la oferta de transporte responde a ellos. La integración logística del transporte dentro de las cadenas de suministro y distribución puede asumirse como una estrategia competitiva de las empresas de manufactura. 
La investigación sobre el tema ha puesto atención en las actividades más dinámicas que acaparan los principales flujos mundiales de comercio: las industrias automotriz y microelectrónica. Además, existe una considerable bibliografía sobre prácticas logísticas dentro de los países centrales, Estados Unidos y Europa principalmente, pero muy poca investigación se ha desarrollado sobre países periféricos.

Como resultado de ese sesgo en la investigación, ocurre fácilmente un error interpretativo cuando se observa el traslape de los flujos internacionales y domésticos en un país periférico, en el sentido de que pareciera que se observa una transición de lo tradicional (prácticas correspondientes al fordismo: sin recurrir a la organización logística) hacia lo globalizado (posfordista: con avanzada organización logística de la producción). El error es similar, e incluso se deriva de la interpretación sin crítica de los seguidores de los llamados "regulacionistas" (Aglietta, 1976, principalmente) en América Latina (Gatto, 1990), a los que el modelo fordista les parece total y tajantemente superado y desplazado por los tipos de organización de la producción llamados por ellos "posfordistas".

Así, para el caso del transporte en México, por ejemplo, la introducción de tecnología de punta en el seguimiento de la carga, el surgimiento de empresas logísticas, la mejora selectiva de la infraestructura carretera y de los ferrocarriles, así como la introducción de prácticas logísticas altamente sofisticadas en algunas empresas, pueden aparecer como señales promisorias de un proceso armónico de modernización que coadyuvan al desarrollo económico, asumiéndose que estas mejoras incrementan las ventajas competitivas de la mayoría de las empresas instaladas en México, en un mundo en feroz competencia.

Otras señales económicas impiden, sin embargo, que esta conclusión valga para el caso mexicano. Ante todo, la mayor parte de la actividad manufacturera -y sobre todo la orientada a la exportación- parece que se dirigiera a la subcontratación de muchos grupos transnacionales, tendencia que ha dado como resultado en México un sistema productivo ampliamente desconectado. Y, segundo, luego de la apertura económica en México (eliminación de las barreras a la importación, liberalización de la inversión extranjera y el Tratado de Libre Comercio de América del Norte, TLCAN), se rompieron las cadenas productivas desarrolladas a lo largo de los 40 años proteccionistas previos y que incorporaban en circuitos, en gran medida endógenos, a las empresas de diferente tamaño. 
Por tanto, y ésta es la premisa de la que aquí se parte, es más certera la observación de los cambios en las actividades económicas en México como un traslape de varios tipos de procesos productivos, algunos de ellos muy avanzados en términos tecnológicos (de acuerdo con los mercados mundializados), mientras otros parecen constreñidos a los marcos organizativos tradicionales, sin mejorar su tecnología, pues sus fracciones de mercado se reducen. No se trata, sin embargo, de la coexistencia separada de varias formas de organización de la producción, ni de maneras separadas de trasladar la mercancía, sino de su combinación necesaria para la readecuación del modelo de acumulación de capital.

Ese desigual desarrollo del sistema productivo en México impide que se generalicen los beneficios de la administración logística, que supuestamente afectarían de manera positiva a todo el sistema económico.

\section{Objetivos}

En el marco de un proyecto de investigación más amplio que persigue explicar la relación entre accesibilidad y prácticas logísticas, por un lado, y la localización industrial, por otro, en el estado de Querétaro, este artículo ofrece analizar los comportamientos logísticos de las plantas manufactureras en esa entidad.

Se eligió Querétaro por varias razones: la principal reconoce el surgimiento en los últimos 15 años de un proceso de industrialización cuya espacialidad recae en algunas de las ciudades medias y otras pequeñas (Ramírez y Arias, 2002), lo que cuestiona las interpretaciones tradicionales sobre el proceso de industrialización, sus hábitos de localización y sus comportamientos de interrelación con otros lugares. Así, en este estudio, los rasgos geográficos de esa región y su dinámica industrial exógena son las principales consideraciones.

No ha escapado a la literatura sobre desarrollo regional y urbano (Delgado, 1998; González y Osorio, 2000 y 2001, entre otros), así como a la referida al transporte de mercancías (González y González, 1990 y 1992), la importancia y peculiaridad de Querétaro en el mapa nacional, lo que explica su dinámica industrial acelerada y en parte correspondiente a las cadenas mundializadas de producción.

Por tanto, el objetivo es doble: por un lado, construir una tipología de firmas manufactureras que pueda dar cuenta de las diferencias en procesos productivos, $y$, por otro, probar que esa tipo- 
logía es adecuada para estudiar las prácticas logísticas en el caso específico de las industrias en Querétaro.

$\mathrm{Al}$ indagar sobre la forma en que las empresas manufactureras seleccionan las empresas que movilizan su mercancía y el control que tienen de su programación, se pretende tener un conocimiento menos ideologizado sobre los comportamientos logísticos en la mundialización que los que se pueden derivar del enfoque regulacionista. Adicionalmente, la indagación sobre la percepción de las empresas manufactureras en relación con las de transporte debe mostrar los resultados de la "externalización" de los requerimientos de la producción mundializada en la esfera de la circulación de mercancías.

Lo anterior se pretende lograr mediante, primero, la construcción de tipos ideales weberianos en los comportamientos logísticos de las empresas cuyas diferencias en tipos de organización de producción deben verse reflejadas en los distintos agrupamientos por rama, tamaño y alcance de mercado. ${ }^{2}$ Posteriormente se contrastan esos tipos ideales con los resultados de una encuesta no estratificada en torno a los comportamientos logísticos. Por último se reinterpretan y reconstruyen los tipos ideales.

\section{Comportamientos logísticos de la manufactura en Querétaro de Arteaga}

El gobierno federal mexicano llevó a cabo una estrategia de desconcentración industrial que perseguía el decremento de la localización industrial de las ciudades más pobladas (principalmente la Ciudad de México), e impulsó la localización industrial en las llamadas ciudades medias, como Santiago de Querétaro.

Adicionalmente, las nuevas plantas manufactureras que querían instalarse en el país buscaron lugares donde los salarios fuesen bajos y el sindicalismo débil, y con infraestructura y servicios de transporte adecuados, y donde la congestión urbana y las demoras fueran mínimas, alejándose así de las grandes conglomeraciones urbanas.

\footnotetext{
${ }^{2}$ Cabe hacer notar que en la bibliografía es común encontrar una correspondencia entre esas dimensiones clasificatorias de las empresas manufactureras. Es así, sobre todo, en cuanto al tamaño y alcance de mercado, al presuponerse que las empresas micro, pequeñas y medianas están asociadas a alcances locales (Alarcón y Stumpo, 2001). En este documento se pretende dar cabida a comportamientos no necesariamente correlacionados entre tamaño, rama y alcance de mercado, al trabajar una matriz compleja de esas dimensiones.
} 
Sobre todo como resultado de estas dos tendencias concurrentes, la actividad industrial creció rápidamente en Querétaro durante las últimas dos décadas (González-Gómez y MartnerPeyrelongue, 1990).

La posición geográfica de ese estado y su posición relativa dentro de las principales redes de transporte terrestre, otorgan a esa región una ventaja competitiva (González-Gómez 1994; González-Gómez y González, 1992) tanto en términos del mercado interno, aún atractivo pero en decremento, como en términos de los flujos comerciales internacionales desde el corazón económico de México hacia Estados Unidos. Esta característica imprime un perfil dual a esa región: como un territorio dedicado a producir partes de la fábrica mundial, bastante similar a las regiones fronterizas mexicanas del norte (González, 2005), y, al mismo tiempo, como un territorio de procesos productivos de alcance doméstico, cercano al comportamiento de las ciudades industriales mexicanas tradicionales.

Finalmente, la actividad industrial del estado se concentra no sólo en su capital, Santiago de Querétaro, sino también a lo largo del corredor industrial que sigue la carretera federal 57, que conecta a la Ciudad de México con la ciudad fronteriza de Nuevo Laredo. Este patrón de localización industrial en corredor está creciendo; su inicio coincidió con la apertura económica del país, desde la segunda mitad de la década de los años ochenta, y fue reforzada después de la puesta en marcha del TLCAN.

Después del TLCAN en 1994, las actividades económicas relacionadas con las cadenas productivas internacionales recibieron un notable impulso. México se convirtió en un lugar muy adecuado para la localización de fragmentos de empresas transnacionales, ya fuera de capital de Norteamérica o de países fuera del subcontinente. Querétaro en particular, ha sido desde entonces también receptor de ese tipo de plantas industriales.

La estrategia industrial del gobierno para atraer capital con el fin de externalizar algunas de sus actividades productivas a países periféricos, ha sido cuestionada desde diferentes perspectivas. Uno de esos cuestionamientos es la duda sobre si la oferta de transporte puede responder eficientemente a las expectativas de calidad de servicio.

Para develar las prácticas logísticas en Querétaro de Arteaga, se consideró necesario analizar las empresas industriales. El gobierno estatal realiza un inventario de las empresas manufactureras. Ese registro abarca todas las unidades económicas que llevan a cabo cualquier tipo de actividad de manufactura. Se 
estratifica el tamaño de la planta, de acuerdo con el Instituto Nacional de Estadística Geografía e Informática (INEGI), en empresas micro, pequeña, mediana y grande (en el territorio queretano no hay las plantas macro). Se realizó una muestra estratificada por tamaño y rama de las 2,388 empresas detectadas en el registro. ${ }^{3}$

Por rama, la estructura muestra una fuerte presencia de la industria de autopartes, aun sin que existan plantas ensambladoras finales de la industria automotriz en el estado o cerca de él. Una de cada tres plantas ubicadas en Querétaro se dedica a esa industria mundializada. La otra presencia fuerte es alimentos, bebidas y tabaco, que puede ser considerada, en términos generales, de alcance local, cuando el tamaño es micro o pequeña y nacional, si es mediana o grande.

La primera preconcepción, que las empresas mundializadas muestran relaciones comerciales de alcance internacional, se valida cuando se observa la respuesta en torno a los flujos internacionales. Alrededor de 58\% de los movimientos internacionales tienen origen o destino en la industria de autopartes (cuadro 1), muy por encima de su participación en la estructura industrial, 29.55\%. Asimismo, esta industria muestra una participación en embarques nacionales que también es mayor que su participación en la estructura industrial, mientras que su participación en embarques locales es algo menor. Lo anterior evidencia no solamente la orientación internacional de la industria de autopartes, sino también una densidad de embarques por empresa mayor que el promedio. Se observa un patrón similar para la metalmecánica, cuya participación en embarques internacionales llega a ser 10 veces mayor que su presencia en términos de número de plantas, y no registra embarques locales. En el caso de Querétaro, estas dos industrias muestran una clara tendencia hacia el alcance internacional (gráfica I)

Sobre las mismas bases se valida la segunda preconcepción; esto es, que las empresas no mundializadas tienen relaciones comerciales principalmente a escala local. Aunque $27.27 \%$ del total de las empresas pertenece a la rama de alimentos, bebidas y

${ }^{3}$ Un coeficiente de correlación Pearson de 0.857 permite definir la muestra como estadísticamente representativa del universo. En el cuestionario se preguntó por la descripción de todos los movimientos de mercancía -de abastecimiento de insumos y de distribución de productos-. Los movimientos se clasificaron de acuerdo con su alcance territorial: internacional cuando el movimiento cruza los límites territoriales de México; nacional cuando cruza los límites estatales, y local cuando ocurre dentro del territorio del estado de Querétaro de Arteaga. 


\section{Cuadro 1}

Distribución de plantas manufactureras y flujos de mercancías en el estado de Querétaro de Arteaga por rama, 1999 (Porcentajes)

\begin{tabular}{lccrr}
\hline \multicolumn{3}{c}{$\begin{array}{c}\text { Número de } \\
\text { plantas }\end{array}$} & & Embarques \\
& & Internacional & Nacional & Local \\
\hline Alimentos, bebidas & & & & \\
y tabaco & 27.27 & 8.89 & 20.77 & 31.03 \\
Textil y del vestido & 4.55 & 2.22 & 0.77 & 2.30 \\
Madera & 11.36 & & 13.08 & 10.34 \\
Química & 9.09 & & 9.23 & 9.20 \\
Hule y plásticos & 4.55 & 2.22 & 1.54 & 10.34 \\
Minerales no metálicos & 9.09 & & 6.92 & 17.24 \\
Autopartes & 29.55 & 57.78 & 31.54 & 18.39 \\
Metalmecánica & 2.27 & 22.22 & 7.69 & \\
Imprenta y edición & 2.27 & 6.67 & 7.69 & 1.15 \\
Total & 100.00 & 100.00 & 100.00 & 100.00 \\
\hline
\end{tabular}

Fuente: Elaboración propia con base en encuesta original.

\section{Gráfica I}

Alcance de embarques por rama (porcentaje)

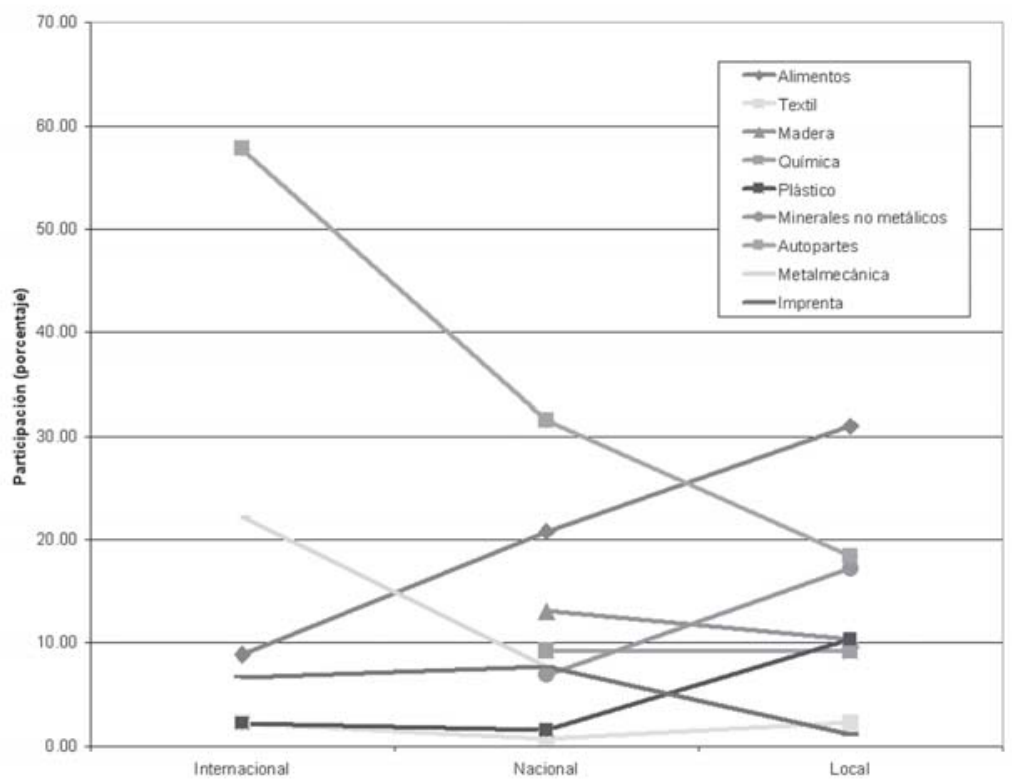

Fuente: Elaboración propia. 
tabaco, sólo 8.89\% de los embarques internacionales corresponden a estas industrias. Se observa que el alcance de mercado de éstas es claramente local. Alcance similar se observa en el caso de minerales no metálicos.

Se derivan cuatro patrones de alcance del análisis de la actividad industrial. El primero, de orientación internacional, muestra una participación en los embarques de carga mayor en el alcance internacional que en el nacional, y éste, en turno, mayor que el local. Este patrón abarca autopartes y metalmecánica.

El segundo patrón muestra el comportamiento opuesto, centrado en movimientos locales principalmente. Este patrón comprende alimentos, bebidas, tabaco y minerales no metálicos. El primero de ellos pertenece, obviamente, al último eslabón de la distribución final, y el segundo está relacionado estrechamente con la industria de la construcción, de alcance local. El tercer patrón, cuyos flujos pertenecen al mercado doméstico (nacional y local), se conforma por madera y química. Y, finalmente, el cuarto no muestra una orientación clara en alcance de mercado, y agrupa las ramas textil y del vestido, hule y plástico, e imprenta y edición.

Cualquier negocio con algún proceso de manufactura está tomado en cuenta en la fuente de información utilizada, y se incluye, por tanto, a muchas empresas cuyas actividades de transporte difícilmente se considerarían de naturaleza logística en la literatura: carpinterías, talleres, tortillerías, panaderías e imprentas. Por esta razón, 75\% de las empresas son micro (cuadro 2) y aproximadamente $15 \%$ son pequeñas; mientras que medianas y grandes son $5 \%$, cada una.

Se detectan dos comportamientos diferenciados en el análisis de las empresas por tamaño. Las empresas micro y pequeñas, tal como sería de esperarse en una región no desarrollada tecnológicamente como Querétaro, hacen negocios principalmente a escala local (gráfica II), como lo detecta Emilio Zeballos (2003: 5557) al analizar las empresas pequeñas y medianas en el ámbito de América Latina. Sin embargo, en Querétaro las empresas medianas tienen comportamientos más cercanos a las grandes, pues están conectadas sobre todo con mercados internacionales.

Al combinar las dos características analizadas, resulta una matriz conceptual de cuatro renglones por alcance (principalmente internacional, indiferenciada, principalmente nacional y principalmente local) y dos columnas por tamaño (grandes y medianas, y pequeñas y micro) (cuadro 3 ). Por el momento, no existen empresas en el estado que pertenezcan a dos de las celdas de esta matriz. 


\section{Cuadro 2}

Distribución de empresas manufactureras y flujos de mercancía en Querétaro de Arteaga por tamaño, 1999 (porcentajes)

\begin{tabular}{|c|c|c|c|c|}
\hline & \multirow[t]{2}{*}{ Empresas } & \multicolumn{3}{|c|}{ Embarques } \\
\hline & & Internacional & Nacional & Local \\
\hline Micro & 75.41 & 13.33 & 26.92 & 45.98 \\
\hline Pequeña & 14.39 & 8.89 & 33.08 & 39.08 \\
\hline Mediana & 4.57 & 33.33 & 13.08 & 5.75 \\
\hline Grande & 5.62 & 44.44 & 26.92 & 9.20 \\
\hline Total & 100.00 & 100.00 & 100.00 & 100.00 \\
\hline
\end{tabular}

Fuente: Elaboración propia con base en encuesta original.

\section{Gráfica 2}

Alcance de embarques por tamaño de planta (porcentaje)

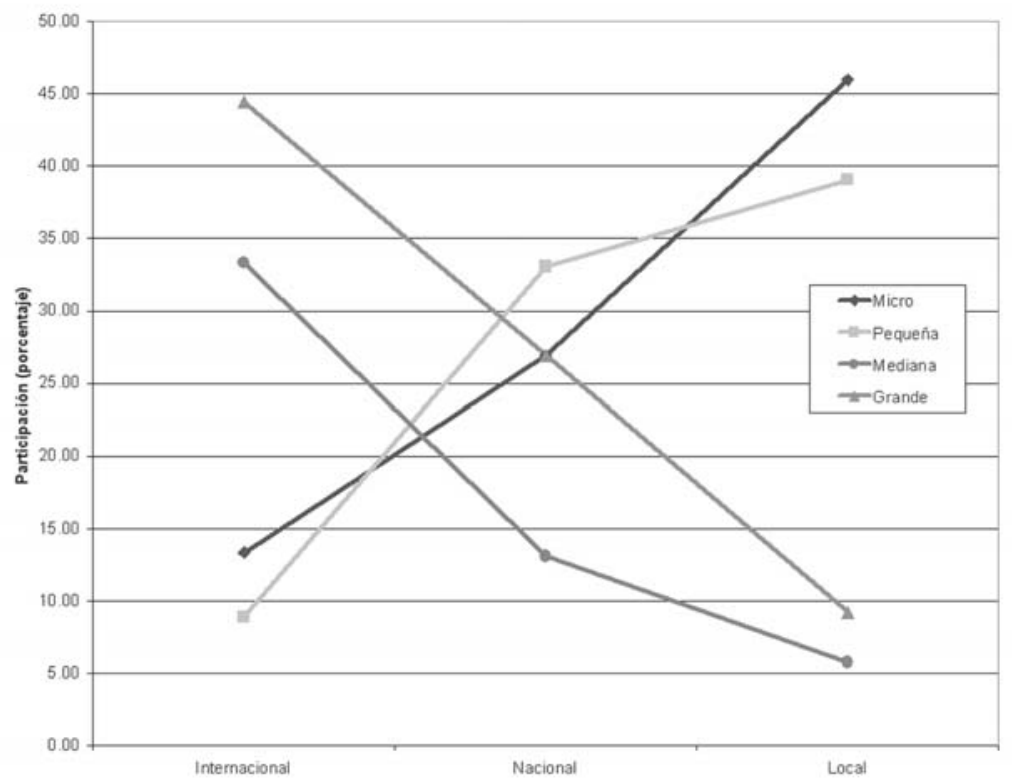

Fuente: Elaboración propia con base en encuesta original.

Con el desarrollo de las prácticas logísticas, las actividades de transporte han sido progresivamente separadas del ámbito directo de la producción. Ello no quiere decir que las empresas manufactureras pierdan el control sobre el transporte. Precisa- 
mente ha ocurrido lo contrario. Una empresa mundializada exitosa puede externalizar sus requerimientos y seleccionar sólo a aquellas empresas de transporte o de servicio logístico que garanticen la calidad necesaria.

Con esto en mente, se pueden construir los comportamientos ideales esperados de los dos extremos de esta tipología. Por un lado, se espera que las empresas grandes y medianas de autopartes y metalmecánica, tipo A, externalicen sus requerimientos de transporte contratando agentes de carga o empresas de transporte de alta tecnología, y prácticamente ninguna actividad de transporte se espera que sea llevada a cabo por la empresa manufacturera.

Por otro lado, se espera que aquellas empresas pequeñas y micro de la industria de alimentos, bebidas y tabaco, o de minerales no metálicos, tipo $H$, realicen por cuenta propia su transportación o contraten empresas de transporte, pero sin externalizar sus requerimientos, así como se ha llevado a cabo esta actividad tradicionalmente.

Se espera que los otros comportamientos logísticos estén entre estos dos extremos. Cuando el alcance de mercado se reduce, el comportamiento logístico debe alejarse del comportamiento logístico idealmente mundializado y acercarse al comportamiento tradicional. Se espera que las empresas pequeñas y micro, $c e-$ teris paribus, tengan un comportamiento tradicional. Se consideran excepcionales, por supuesto, aquellas empresas pequeñas (incluso micro) asociadas con procesos productivos mundializados. Cabe mencionar que esta diferenciación se aborda poco en la bibliografía reciente sobre las pequeñas y medianas empresas (Pymes) pues la mayor parte de los autores ven el tamaño directamente asociado con el alcance de mercado y, en ese sentido, las Pymes son consideradas como pertenecientes a los procesos endógenos e incluso locales (Zeballos, 2003: 54)

\section{Selección de empresas transportistas}

Se preguntó a los administradores encuestados no sólo acerca de las características geográficas de los flujos de mercancía, sino también sobre el actor específico que maneja la carga o es responsable del embarque. Se clasificó a los actores por tipo (agentes de carga, transportistas y otros no transportistas) y, en el caso de las empresas de transporte, también por nacionalidad y tamaño. 


\section{Cuadro 3 \\ Tipología de comportamiento logístico}

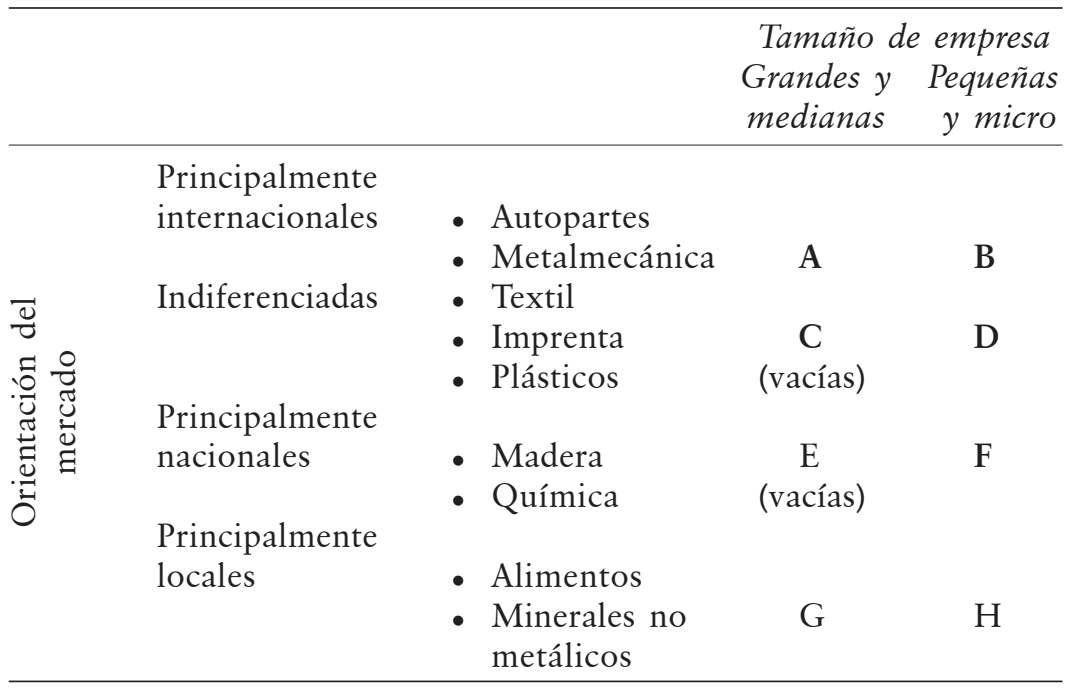

Fuente: Elaboración propia.

Los datos recabados parecen validar la tipología anterior. Las empresas tipo A, internacionales en orientación de mercado y relativamente grandes, para resolver sus servicios internacionales recurren en lo fundamental a un agente de carga o a una empresa transportista de alta calidad de servicio, 90.64\% (cuadro 4), y ninguno de los embarques lo realiza la propia empresa de manufactura.

Deben resaltarse dos características más de ese comportamiento. Los embarques de alcance nacional no los hacen principalmente empresas grandes y, por tanto, tecnológicamente avanzadas, como sería de esperarse, sino pequeñas empresas mexicanas. La explicación para esta aparente discrepancia reside en las características del mercado doméstico de transporte en México, dominado en precio y condiciones claramente por los consumidores de este servicio desde la desregulación de 1989, a partir de la cual las tarifas no están controladas ni se otorgan concesiones de exclusividad por ruta, lo que ha llevado a una aguda competencia entre empresas transportistas y a un mercado dominado por los clientes. Uno de los gerentes entrevistados afirma que prefiere para este tipo de viajes a las empresas de tamaño mediano porque, aunque sus características tecnológicas no sean las óptimas, proveen un alto nivel de servicio ante el 
riesgo de perder los contratos subsiguientes, y el precio con ellas puede ser negociado en condiciones ventajosas.

La otra característica notable aquí es la presencia de la organización de la producción de tipo cliente-proveedor, también a partir del proceso de mundialización. Clientes o proveedores fueron reportados por los encuestados como responsables de una parte no trivial de los embarques internacionales (6.25\%), una pequeña parte de los nacionales $(4.88 \%)$ y una relativamente grande parte de los embarques locales (50\%). Esto no quiere decir que clientes o proveedores por sí mismos realicen esta actividad; con seguridad contratan empresas transportistas para ello. Pero el punto es que ellos son responsables del control sobre esta actividad y el entrevistado no está al tanto de su resolución. Este hecho muestra una de las características del esquema cliente-proveedor, donde cada planta es responsable de sólo una parte muy claramente definida del proceso productivo en su conjunto.

Las empresas pequeñas y medianas de autopartes y metalmecánicas, tipo B, muestran un comportamiento polarizado. Confían básicamente en empresas transportistas grandes para sus embarques internacionales, mientras realizan por sí mismas todos los embarques nacionales y locales. La presión que ejerce la industria automotriz en estas empresas las obliga a estar muy al pendiente de la oportunidad en la entrega. Sin embargo, por su tamaño, y para no perder el control sobre los estándares de entrega, contratan empresas de alta tecnología o llevan a cabo por sí mismas el transporte.

Para adecuarse a estos requerimientos de calidad y a las expectativas de entrega, dentro de las limitantes de presupuesto de estas empresas, el único porteador disponible para ellas es el servicio de paquetería.

En Querétaro, las empresas pequeñas y micro de las ramas textil, imprenta y plásticos muestran una tendencia de integración al proceso de mundialización. Por esta razón, estas empresas, tipo $\mathrm{D}$, tienen un comportamiento logístico bastante similar al de las tipo B.

Consideramos aquí que esto es sólo una tendencia, pues existen diferencias significativas con las empresas tipo B. Los embarques internacionales se distribuyen equitativamente entre los diferentes tipos de porteadores, los servicios de paquetería son los principales porteadores de los embarques nacionales, y los movimientos locales los hace la propia empresa.

Las empresas grandes y medianas de las ramas alimentos y minerales no metálicos, agrupadas en el tipo $G$, parecen tener ya 


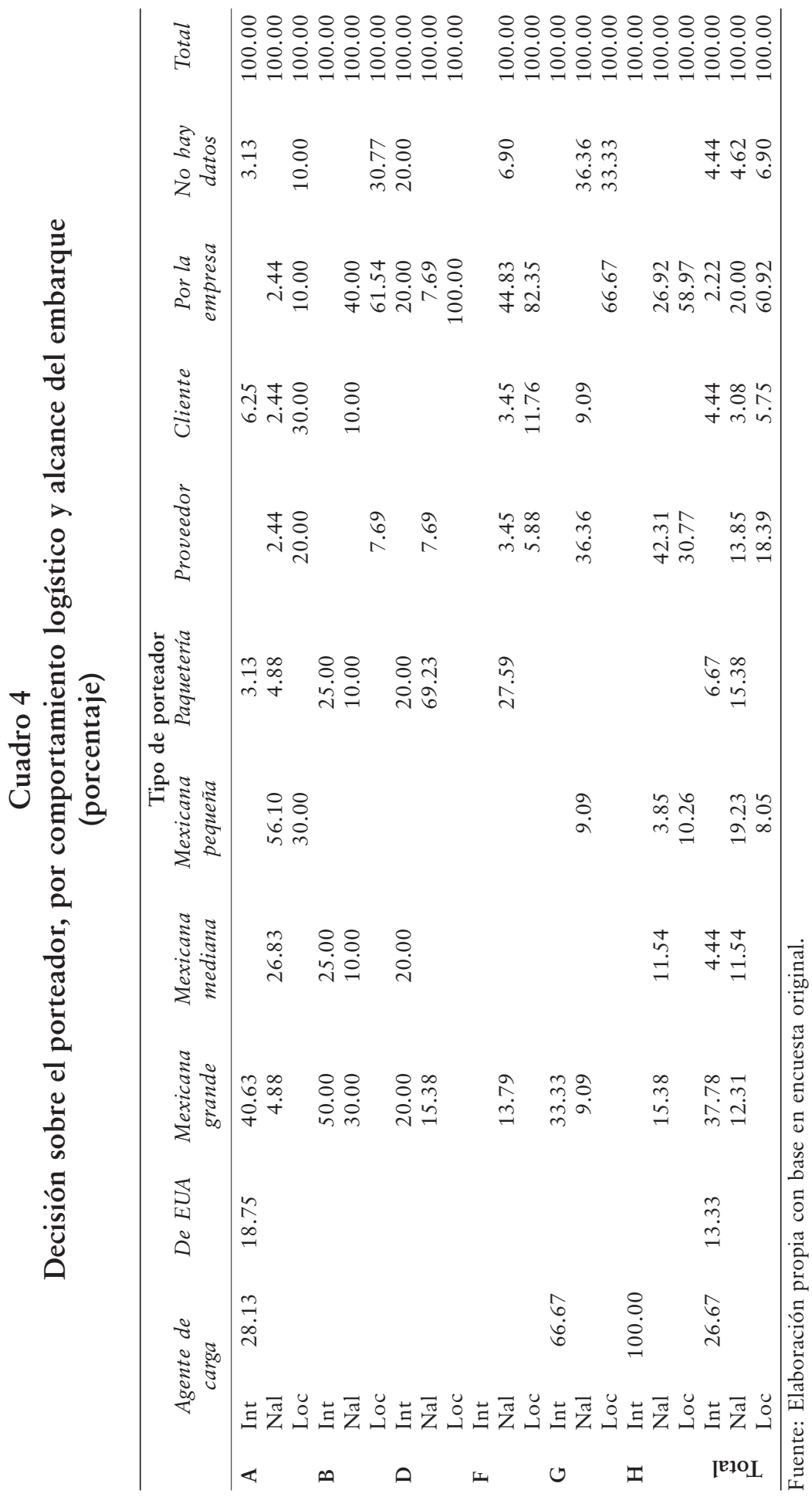


asumidos los modernos procedimientos logísticos, recurriendo fuertemente al esquema cliente-proveedor para los embarques nacionales y a las empresas de alta tecnología para los internacionales. Al mismo tiempo, sin embargo, la mayoría de los embarques locales los realiza la propia empresa.

Finalmente, las empresas pequeñas y micro de las ramas anteriores, tipo $\mathrm{H}$, no muestran el mismo comportamiento logístico descrito antes. A pesar de que una proporción muy grande de los embarques locales (los más recurrentes en este grupo) los hace la misma empresa, algunos pocos internacionales se resuelven contratando un agente de carga. Adicionalmente, los proveedores son responsables de la mayor parte de los viajes de alcance nacional. El primer rasgo observado concuerda con un comportamiento "tradicional", mientras lo otros dos concuerdan más con una administración logística "moderna".

\section{Percepción de la industria sobre el desempeño de los proveedores de transporte}

La competencia en el mercado de transporte, surgida a partir de las profundas transformaciones en el marco regulatorio mexicano después de 1989, produjo una diferenciación en la calidad de servicio que ha segmentado la oferta.

El cambio tecnológico hacia la modernización del equipo y hacia la mejora en técnicas de rastreo de los embarques, de ruteo y de programación de entregas no ha alcanzado, sin embargo, todo el abanico de la oferta de servicios de transporte en México. De hecho, sólo una pequeña proporción de las empresas de transporte puede ser considerada con estándares internacionales de calidad.

De esta forma, los varios segmentos de la oferta de transporte se dedican a atender los diversos requerimientos de la demanda. Sin embargo, las expectativas en calidad de servicio por parte de la demanda de traslado de la mercancía han alcanzado la mayor parte de los segmentos de demanda.

Por otro lado, las empresas de manufactura de carácter mundial contratan no sólo empresas de transporte de alta tecnología; para sus múltiples embarques nacionales o locales contratan las empresas disponibles en el mercado.

La carencia de empresas de transporte con alta calidad de servicio en el ámbito local y la persistencia de empresas de manufactura de alcances local y nacional que no tienen altos reque- 


\section{Cuadro 5}

Promedio de calificaciones sobre los atributos del embarque, por rama de actividad

\begin{tabular}{|c|c|c|c|c|c|c|c|c|}
\hline & & $A$ & $B$ & $D$ & $F$ & $G$ & $H$ & Total \\
\hline \multirow[t]{3}{*}{ Costo } & Int & 8.33 & 8.00 & 6.00 & & 8.00 & 9.00 & 7.92 \\
\hline & $\mathrm{Nal}$ & 8.33 & 10.00 & 6.75 & 9.60 & 8.00 & 8.50 & 8.48 \\
\hline & Loc & 9.67 & 10.00 & 4.50 & 9.50 & 8.00 & 9.40 & 8.96 \\
\hline \multirow[t]{3}{*}{ Tiempo } & Int & 7.83 & 8.67 & 7.00 & & 9.00 & 10.00 & 8.07 \\
\hline & $\mathrm{Nal}$ & 7.83 & 9.00 & 6.25 & 8.75 & 9.00 & 7.33 & 7.79 \\
\hline & Loc & 8.33 & 9.00 & 4.00 & 8.67 & 9.50 & 9.10 & 8.50 \\
\hline \multirow[t]{3}{*}{ Disponibilidad } & Int & 9.17 & 7.00 & 8.33 & & 8.00 & 9.00 & 8.54 \\
\hline & $\mathrm{Nal}$ & 9.33 & 10.00 & 8.25 & 9.75 & 9.50 & 8.50 & 9.08 \\
\hline & Loc & 9.00 & 9.00 & 6.00 & 9.00 & 9.50 & 9.30 & 8.91 \\
\hline \multirow[t]{3}{*}{ Confiabilidad } & Int & 8.17 & 8.33 & 7.67 & & 8.00 & 10.00 & 8.21 \\
\hline & $\mathrm{Nal}$ & 8.00 & 10.00 & 7.75 & 8.50 & 8.50 & 9.33 & 8.58 \\
\hline & Loc & 8.67 & 4.50 & 7.00 & 8.00 & 9.50 & 8.40 & 8.00 \\
\hline \multirow{3}{*}{$\begin{array}{l}\text { Seguimiento de la } \\
\text { carga }\end{array}$} & Int & 8.17 & 8.50 & 7.33 & & 9.00 & 8.00 & 8.08 \\
\hline & $\mathrm{Nal}$ & 8.17 & 5.00 & 7.25 & 7.25 & 7.00 & 5.67 & 6.87 \\
\hline & Loc & 8.00 & 4.00 & 6.00 & 6.67 & 10.00 & 4.33 & 5.65 \\
\hline \multirow{6}{*}{$\begin{array}{l}\text { Tamaño de la } \\
\text { empresa de } \\
\text { transporte } \\
\text { Condiciones físicas } \\
\text { del equipo }\end{array}$} & Int & 7.33 & 10.00 & 8.50 & & 10.00 & 5.00 & 7.82 \\
\hline & $\mathrm{Nal}$ & 7.50 & 5.00 & 6.50 & 6.00 & 5.50 & 4.00 & 5.83 \\
\hline & Loc & 8.00 & 4.00 & 3.00 & 6.67 & 7.50 & 3.60 & 5.05 \\
\hline & Int & 9.00 & 6.50 & 8.67 & & 8.00 & 8.00 & 8.38 \\
\hline & $\mathrm{Nal}$ & 9.17 & 4.00 & 7.00 & 7.25 & 9.00 & 6.67 & 7.42 \\
\hline & Loc & 9.33 & 4.00 & 6.50 & 7.33 & 9.50 & 7.10 & 7.32 \\
\hline
\end{tabular}

Fuente: Elaboración propia con base en encuesta original.

rimientos tecnológicos para sus traslados, desde el punto de vista económico, pero que están conscientes de las diferencias en las características de calidad en el servicio, resultan de la hipótesis de que las empresas tipo A debieran estar satisfechas con el desempeño de la oferta de alcance internacional, pero insatisfechas con la respuesta de la oferta de transporte local e incluso nacional. En el otro extremo de la tipología, las empresas tipo $\mathrm{H}$ no estarían atentas al desempeño de las empresas de transporte de alcance internacional y debieran estar satisfechas con la respuesta local.

Esta hipótesis no se confirma en las calificaciones que dieron los gerentes de las empresas de manufactura (de 0 a 10) sobre diferentes atributos del desempeño real de la oferta de transporte utilizada (cuadro 5). En general, las calificaciones son bastante altas, lo que expresaría un desempeño satisfactorio, con la única excepción de las calificaciones otorgadas por las empresas tipo D. 
Sin embargo, un análisis más profundo de los resultados parece apoyar una diferencia más compleja en la percepción sobre el desempeño de la oferta.

No debe considerarse el costo como un atributo de la oferta -más adecuadamente es la expresión económica de la manera en que se resuelve el mercado-. Ciertamente, las empresas que asumen tener un sistema logístico valoran los sistemas de transporte más en términos de sus atributos que de su costo. Buscan un oferente que provea no sólo servicios específicos, sino también que cumpla sus requerimientos en tiempo, frecuencia y disponibilidad.

$\mathrm{El}$ costo de los embarques internacionales lo califican como adecuado las empresas tipo A, B, G y H. Muy probablemente, la calificación promedio está sesgada por los valores de las industrias más representativas: de autopartes y de alimentos y bebidas, que ya han adquirido suficiente experiencia y poder en la negociación de los embarques internacionales, independientemente del tamaño de la empresa. Otras ramas en Querétaro, incluidas en las de tipo D y G, no hacen uso de embarques internacionales o lo consideran muy costoso.

En general, los otros dos alcances de embarques, nacionales y locales, recibieron buenas calificaciones en costo. Se puede notar, también, que las empresas dentro de los tipos $\mathrm{B}$ y $\mathrm{H}$ otorgaron mejores calificaciones que las de los tipos A y G, que comprenden, respectivamente, las mismas ramas, lo que muestra la mayor preocupación de las grandes y medianas por sus estructuras de costo.

Se mantiene la misma conclusión para las empresas tipo F. La única excepción notable, de nuevo, es el tipo D. No se puede construir una explicación para esta diferencia con los datos recabados, y se requeriría mayor investigación en este tema específico.

Los atributos relevantes del desempeño considerados aquí son tiempo, disponibilidad, confiabilidad, seguimiento de la carga y condición física del equipo.

Se observa un patrón en las calificaciones para seguimiento de la carga y condición física del equipo, en el cual las empresas tipos A y $\mathrm{G}$ otorgaron mayores calificaciones a ambos atributos, mientras que las tipo $\mathrm{B}$ y $\mathrm{H}$-micro y pequeñas- las califican entre regular y pobre. Esto sugiere que algunas compañías de transporte han logrado condiciones adecuadas en estos atributos para cumplir con los requerimientos mundializados de las empresas grandes, mientras que el servicio ofrecido por las compañías de alcances nacional y local se maneja todavía en una forma tradicional, con pobre desempeño. 
No se puede detectar un comportamiento consistente para las calificaciones sobre tiempo, que es una característica en gran medida fuera del control del desempeño de las empresas de transporte y depende más de la infraestructura para el transporte. Tampoco pueden delinearse diferencias entre las calificaciones para disponibilidad, confiabilidad y tamaño de la firma.

\section{Conclusiones}

Parece haber suficiente evidencia para apoyar la hipótesis de que la introducción de las prácticas mundializadas en la manufactura localizada en Querétaro de Arteaga ha conducido al desarrollo de varios requerimientos logísticos diferentes que muestran comportamientos logísticos diversos. Esta conclusión apoya, entonces, una interpretación de yuxtaposición de diferentes formas de organización de la producción en la planta manufacturera instalada en México, más que un recambio a la manera de la interpretación regulacionista sobre el "posfordismo".

En este artículo se muestran algunas características de tales comportamientos diversos. La tipología propuesta parece ser útil para analizar prácticas logísticas y da algunas pistas que indican cómo la oferta actual de transporte está segmentada, no sólo en términos de precio sino también en cuanto a calidad de servicio.

La otra conclusión importante es que la industria muestra comportamientos logísticos diversos como resultado de la combinación de dos variables: tamaño de la empresa y rama de actividad. Por tanto, la oferta se segmenta como respuesta a esta diferenciación en la demanda.

Sin embargo, los límites entre comportamientos logísticos no son nítidos. Así, los consumidores de transporte, las empresas de manufactura, no contratan empresas sólo de su segmento esperado, sino también de algunos otros.

Finalmente, todavía es verdad (como énfasis) que las empresas grandes despliegan mayores alcances de mercado en sus relaciones productivas, y que las pequeñas están más orientadas hacia el mercado local. El alcance de las relaciones económicas exhibe una coincidencia notable con las características tecnológicas de las empresas de transporte, mientras más amplio es el alcance, mayor tecnología le corresponde. Sin embargo, la aparición de empresas pequeñas, e inclusive micro, que interactúan en las cadenas productivas mundiales distorsiona, hasta cierto grado, esta simple dicotomía. 


\section{Bibliografía}

Aglietta, Michel (1976), Regulación y crisis del capitalismo, Siglo XxI, México.

Alarcón, Cecilia y Giovanni Stumpo (2001), "Políticas para pequeñas y medianas empresas en Chile", Revista de la CEPAL, Santiago de Chile, agosto, 74: 175-194.

Antún Callaba, Juan Pablo (1995), Logística: una visión sistémi$c a$, IMT San Fandila, México.

Bowersox, Donald y David Closs (1996), Logistical Management. The Integrated Supply Chain Process, McGraw-Hill, Nueva York.

Delgado, Javier (1998), Ciudad-región y transporte en el México central, un largo camino de rupturas y continuidades (1998), Instituto de Geografía y Programa Universitario de Estudios sobre la Ciudad, Universidad Nacional Autónoma de México, México.

Fröbel, Folker, Jürgen Hienrichs y Otto Kreye (1977), The New International Division of Labor, Siglo XXI, México.

Gatto, Francisco (1990), “Cambio tecnológico neofordista y reorganización productiva. Primeras reflexiones sobre sus implicaciones territoriales", en Francisco Alburquerque Llorens, Carlos A. de Matos y Ricardo Jordán Fuchs (coords.), Revolución tecnológica y reestructuración productiva: impactos y desafíos territoriales, ILPES/ONU, Buenos Aires, pp. 55-102.

González Gómez Carmen Imelda y Lorena Osorio (2000), Cien años de industria en Querétaro, Universidad Autónoma de Querétaro-Secretaría de Desarrollo Sustentable, Gobierno del Estado, Querétaro.

González Gómez Carmen Imelda y Lorena Osorio (2001), La industria en Querétaro, polarización y desarrollo, UAQ-SUPauaq. México. 
González-Gómez, Ovidio (2005), Territorio de la producción mundial, la Frontera Norte, Universidad Autónoma de Querétaro, Querétaro, México.

González-Gómez, Ovidio y Carlos Martner-Peyrelongue (1990), "Querétaro, ciudades fragmentadas”, Ciudades, Red Nacional de Investigación Urbana, México, abril-junio, 6: 23-30.

González-Gómez, Ovidio y Carmen Imelda González Gómez (1990), Transporte en Querétaro en el Siglo XIX, Instituto Mexicano del Transporte-Gobierno del Estado de Querétaro, México.

González-Gómez, Ovidio y Carmen Imelda González Gómez (1992), Transporte en Querétaro en el Siglo XX, surgimiento y desarrollo del autotransporte, Instituto Mexicano del Transporte-Gobierno del Estado de Querétaro, México.

González-Gómez, Ovidio et al., (1994), Subsistema de ciudades en el estado de Querétaro, Consejo Estatal de Población, Querétaro, México.

Piore, Michael y Charles Sabel (1984), The Second Industrial Divide: Possibilities for Prosperity, Basic Books, Nueva York.

Ramírez, Blanca y Patricia Arias (2002), "Hacia una nueva rusticidad”, Ciudades, Puebla, México, abril-junio, 54: 9-14.

Tixier, Daniel, Hervè Mathe y Jacques Colin (1988), La logistique au service de l'entreprise, Dunod, París.

Wallerstein, Immanuel (1979), The Capitalist World Economy, Cambridge University Press, Cambridge.

Zevallos, Emilio (2003), “Micro, pequeñas y medianas empresas en América Latina", Revista de la CEPAL, Santiago de Chile, abril, 79: 53-70. 
Recibido: 24 de febrero de 2006.

Reenviado: 29 de mayo de 2006. Reenviado: 28 de septiembre de 2006. Aprobado: 9 de octubre de 2006.

Ovidio González Gómez es doctor en ciencias sociales por la Universidad de Guadalajara, asimismo realizó sus estudios de Arquitectura en el Instituto Politécnico Nacional y de maestría en Planeación Urbana y Regional en la Universidad de Illinois. Actualmente es investigador nivel I en el Sistema Nacional de Investigadores (SNI) y es el coordinador de la Maestría en Sistemas de Transporte y Distribución de Carga de la Universidad Autónoma de Querétaro. Sus líneas de investigación actuales son desarrollo regional y transporte y comercio internacional y transporte. Entre sus publicaciones destacan: Frontera México-Estados Unidos en la economía Mundo, Revista de La Economía Internacional, del Centro de Investigaciones en Economía Internacional, Universidad de La Habana, marzo 2007, número 4; "Mercados segmentados de transporte en la Región CentroNorte, QUIVERA”, noviembre 2000, año 2, número 4, pp. 37-58 y "Territorio flexible en la semiperiferia. La frontera norte mexicana", EURE, número 74, 1999: Pontificia Universidad Católica de Chile.

Rossana Nieto López es maestra en Sistemas de Transporte y Distribución de Carga por la Universidad Autónoma de Querétaro (UAQ), asimismo realizó sus estudios de sociología en la propia UAQ. Actualmente es profesora en el Colegio de Bachilleres del Estado de Querétaro (COBAQ). 